\title{
Elément de poutre multicouche avec glissement d'interface
}

\author{
Blaise Rebora — François Frey \\ LSC - Laboratoire de Mécanique des Structures et Milieux Continus \\ EPFL - Ecole Polytechnique Fédérale de Lausanne \\ Station 18 - CH-1015 Lausanne \\ \{blaise.rebora, francois.frey\}@epfl.ch
}

\begin{abstract}
RÉSUMÉ. Cet article présente un élément multicouche de poutre plane à deux nœuds, droit ou courbe, de type Bernoulli, à nombre de couches arbitraire, avec glissement aux interfaces. Les couches et leurs interfaces suivent des lois constitutives non linéaires. Les grands déplacements sont traités par la cinématique non linéaire de von Karman couplée à la formulation lagrangienne corotationnelle des déplacements; les déformations et les glissements restent petits et les grandes rotations résultent du cumul de rotations modérées. L'élément est exempt de verrouillage. On en illustre et valide le potentiel à travers des tests variés.

ABSTRACT. This paper presents a multilayered two node planar beam element, straight or shallow, of Bernoulli type, with an arbitrary number of layers with interlayer slip. Material and geometric nonlinearities are included. Small strains and slips are assumed. Large displacements are dealt with von Karman strain coupled with corotational formulation. No locking appears. Various tests show the capabilities of this element.

MOTS-CLÉS: poutre multicouche, glissement d'interface, grands déplacements, formulation corotationnelle, matériaux non linéaires.

KEYWORDS: multilayered beam, interlayer slip, large displacements, corotational formulation, material nonlinearity.
\end{abstract}

DOI:10.3166/REMN.16.559-581 @ Lavoisier, Paris 


\section{Introduction}

Un effort de recherche considérable a été entrepris, ces vingt dernières années, dans l'analyse des structures multicouches (poutres, plaques et coques). Les articles de synthèse (Kapania et al., 1989; Noor et al., 1996) et (Carrera, 2001, 2002) donnent plus de 2000 références. Pour la plupart, elles se limitent au domaine élastique linéaire. Dans le domaine géométriquement non linéaire, les développements analytiques sont peu nombreux et restreints aux rotations modérées (type von Karman) ; les grands déplacements sont abordés dans quelques articles via les méthodes numériques. La non-linéarité matérielle est rarement traitée, car les applications pratiques concernent avant tout les matériaux composites (verre/aramide/carbone).

Les couches sont généralement admises parfaitement liées : les interfaces sont rigides et infiniment minces. Si la rupture des interfaces (délamination) fait l'objet de recherches intenses actuellement, l'étude des multicouches avec glissement aux interfaces (interlayer slip - sans séparation transversale des couches) reste un sujet confidentiel, où de surcroît le comportement de l'interface est ordinairement admis élastique linéaire.

Or, dans de nombreux domaines (génie civil, mécanique, aéronautique), les interfaces sont déformables, et ce d'autant plus qu'on se rapproche de l'état de ruine. C'est le cas par exemple pour les poutres lamellées clouées ou vissées en bois (en 1922 déjà, (Engesser, 1922) aborde le problème des poutres en bois chevillées avec glissement d'interface), le verre structurel laminé, les ressorts à lames ou les poutres mixtes acier-béton. Vu les caractéristiques très variables des couches, on doit utiliser une théorie à couches discrètes (discrete layer theory); les théories à couche équivalente unique (equivalent single layer theory), à couches multiples de type zigzag (multilayer zigzag theory) ou de type Reissner mixte (mixed Reissner theory) ne conviennent pas.

L'équation différentielle traduisant le comportement flexionnel linéaire d'une poutre, de type Bernoulli, formée de deux couches avec glissement d'interface est donnée pour la première fois par (Stüssi, 1943), dans le cadre de la déformation de cisaillement des rivets d'une poutre en acier, à âme pleine, composée rivée. Suivent des applications aux poutres en bois chevillées et aux poutres mixtes acier-béton (Stüssi, 1947). Un développement identique est publié par (Newmark et al., 1951) pour les poutres mixtes acier-béton.

Le problème est repris par les écoles d'expression allemande dès les années 1950, tant pour le bois que pour le mixte ; on trouvera les références-clés dans (Moehler, 1956), reprises par (Pischl, 1968). La recherche est particulièrement active dans le domaine des poutres en bois faites de deux couches (ou trois couches avec disposition symétrique): partant des travaux de (Moehler, 1956) et des solutions analytiques de cas simples couvrant la flexion (type Bernoulli) et le flambement d'Euler, on propose une méthode générale de dimensionnement 
utilisable en pratique, la méthode $\gamma$, qui entre dans certaines normes (voir (Heimeshoff, 1987)). Schelling (1968) généralise la solution analytique, puis la méthode $\gamma$, à un nombre quelconque de couches. A l'occasion d'une recherche sur les poutres mixtes béton-bois, une excellente synthèse est dressée par (Natterer et al., 1987). Plus récemment, une nouvelle méthode pratique issue des recherches de (Kreuzinger, 1995), dite de l'analogie du cisaillement, est proposée. On trouve, dans les travaux récents de (Frangi et al., 2003) et (Pirazzi, 2005), des commentaires sur ces deux méthodes.

Dès 1968 apparaît, aux USA, une série d'articles sur les poutres en bois multicouches avec glissement. Dans (Goodman et al., 1968) et (Vanderbilt et al., 1974), on reprend la solution analytique de (Newmark et al., 1951) et on la résout en tenant compte d'une loi de glissement d'interface non linéaire; dans (Thompson et al., 1975) on propose une solution numérique par éléments finis. On est en géométrie linéaire, théorie de Bernoulli et poutre à deux couches (ou trois symétriquement disposées). Les développements analytiques sont aussi appliqués à la poutre sandwich, avec recherche de la charge critique d'Euler (Fazio et al., 1982), puis plus tard ils sont étendus à l'analyse de second ordre linéarisé (Kamiya, 1987 ; Girhammar et al., 1993 ; Wheat et al., 1994).

Ce n'est que récemment qu'un élément fini de poutre à nombre quelconque de couches est proposé, incluant le second ordre linéarisé (Gollwitzer et al., 2003) ; les applications toutefois se limitent au premier ordre. Un élément très général, matériellement et géométriquement non linéaire, à deux couches cependant, est ensuite publié par (Cas et al., 2004), mais les exemples restent dans le domaine des rotations modérées.

\section{But}

On présente ici un élément de poutre plane à deux nœuds, droit ou courbe, de type Bernoulli, à nombre $N$ de couches arbitraire, avec glissement entre les couches (mais sans séparation des couches), à matériau non linéaire tant dans les couches qu'aux interfaces, et à cinématique non linéaire (en rotations modérées, mais en petites déformations). Le champ de déplacements est transversalement cubique (flexion fonctions de Hermite) et axialement quadratique; le degré de liberté central axial de chaque couche permet d'éviter de façon optimale tous les verrouillages de l'élément de Bernoulli (Frey et al., 1992) et est condensé statiquement avant assemblage. Chaque noud possède $N+2$ inconnues déplacements ( $N$ déplacements axiaux, un déplacement transversal et une rotation). L'élément peut être initialement légèrement courbe (shallow) par l'emploi de la déformation de Marguerre. Les grands déplacements sont traités par la cinématique de von Karman couplée à la formulation lagrangienne corotationnelle des déplacements. La non-linéarité matérielle est unidimensionnelle tant pour les couches (loi $\sigma-\varepsilon$ arbitraire) que pour les interfaces (loi cisaillement-glissement quelconque). 
Un élément de mêmes caractéristiques est proposé dans (Krawczyk et al., 2006), mais où les couches obéissent à la théorie des poutres de Timoshenko (Batoz et al., 1990). Chaque nœud a plus d'inconnues, à savoir $2 N+1$, mais l'élément a l'avantage de tenir compte de la déformation d'effort tranchant et de livrer les contraintes tangentielles dans les couches.

\section{Forme forte linéaire}

La section droite d'une poutre plane multicouche, rectiligne et prismatique, est composée de $N$ rectangles (figure 1) ; pour chaque rectangle $i(i=1, N)$, on a : largeur $b_{i}$, épaisseur $t_{i}$, aire $A_{i}\left(=b_{i} t_{i}\right)$, inertie $I_{i}\left(=b_{i} t_{i}{ }^{3} / 12\right)$ et module de Young du matériau $E_{i}$; pour chaque interface $i, i+1(i=1, N-1)$, on a un module de glissement $k_{i, i+1}\left[\mathrm{~F} / \mathrm{L}^{2}\right]$ associé au glissement relatif $g_{i, i+1}[\mathrm{~L}]$ des couches $i$ et $i+1$ (petit glissement, mesuré en configuration déformée). En long, la poutre est rapportée aux axes $(x, y)$, où $x$ coïncide avec l'axe de la couche 1 (on généralise sans peine pour une position quelconque de $x$ ) ; le déplacement axial de chaque couche est $u_{i}$; le déplacement transversal commun (pas de décollement) est $v$ et la rotation commune (poutre de Bernoulli) est $v^{\prime}=d v / d x$ (l'indice prime indique la dérivée).

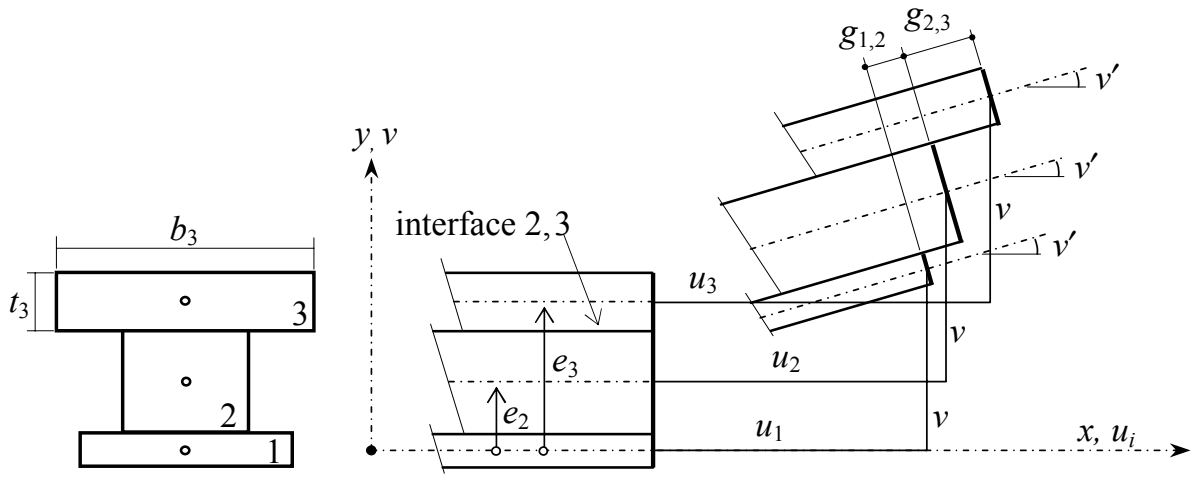

Figure 1. Poutre multicouche : section, configurations initiale et déformée

Isolant un tronçon $d x$ de la couche $i$, on exprime son équilibre en fonction des déplacements. On appelle $N_{i}, V_{i}$ et $M_{i}$ les efforts intérieurs (efforts normal et tranchant, moment de flexion), $n_{i}$ et $q_{i}$ les charges extérieures, axiale et transversale, réparties à l'axe de la couche, et enfin $f_{i, i+1}$ et $p_{i, i+1}$ les forces, tangentielle et normale, réparties aux interfaces (action d'une couche sur l'autre).

Pour les seuls effets axiaux (ou membranaires), on a (figure 2)

$$
\sum F_{x}=0 \quad \Rightarrow \quad N_{i}^{\prime}+f_{i, i+1}-f_{i-1, i}+n_{i}=0
$$


En dérivant la loi constitutive $N_{i}=E_{i} A_{i} u_{i}^{\prime}$ pour l'introduire dans l'équation précédente, on obtient (où $u_{i}$ est, pour rappel, le déplacement axial au niveau de la ligne moyenne de la couche $i$ )

$$
E_{i} A_{i} u_{i}^{\prime \prime}+f_{i, i+1}-f_{i-1, i}+n_{i}=0
$$
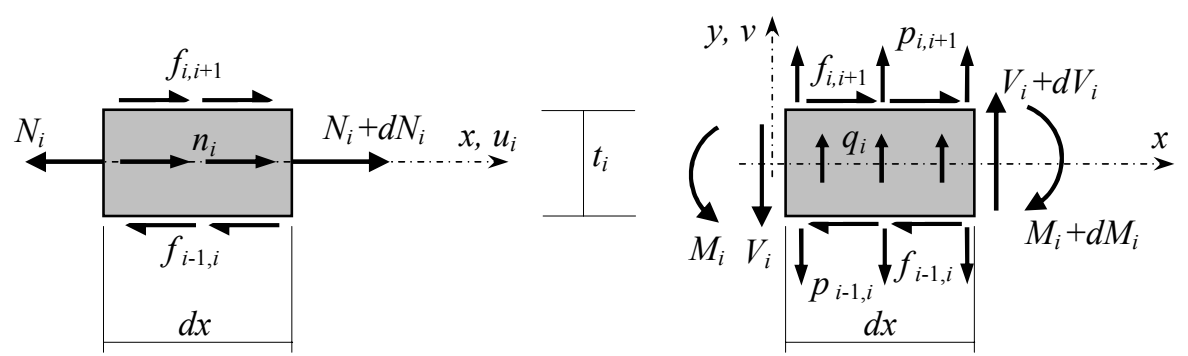

Figure 2. Forces intervenant dans la sollicitation axiale

Figure 3. Forces intervenant dans la sollicitation transversale

Pour les seuls effets transversaux (ou flexionnels), on a (figure 3)

$$
\begin{aligned}
& \sum F_{y}=0 \quad \Rightarrow \quad V_{i}^{\prime}+p_{i, i+1}-p_{i-1, i}+q_{i}=0 \\
& \sum M=0 \quad \Rightarrow \quad-M_{i}^{\prime}+V_{i}-\frac{1}{2} t_{i}\left(f_{i, i+1}+f_{i-1, i}\right)=0
\end{aligned}
$$

En dérivant la loi constitutive $M_{i}=-E_{i} I_{i} v^{\prime \prime}$ pour l'insérer dans l'équation d'équilibre en rotation, on obtient :

$$
V_{i}=-E_{i} I_{i} v^{\prime \prime \prime}+\frac{1}{2} t_{i}\left(f_{i, i+1}+f_{i-1, i}\right)
$$

puis on dérive cette équation pour l'introduire dans l'équation d'équilibre selon $y$

$$
-E_{i} I_{i} v^{\prime \prime \prime \prime}+\frac{1}{2} t_{i}\left(f_{i, i+1}+f_{i-1, i}\right)^{\prime}+p_{i, i+1}-p_{i-1, i}+q_{i}=0
$$

Aux interfaces, la loi de glissement est $(i=1, N-1)$

$$
f_{i, i+1}=k_{i, i+1} g_{i, i+1}
$$

On peut exprimer le glissement en fonction des déplacements $u_{i}$ et $v$ (figure 1)

$$
g_{i, i+1}=\left(u_{i+1}+\frac{1}{2} t_{i+1} v^{\prime}\right)-\left(u_{i}-\frac{1}{2} t_{i} v^{\prime}\right)
$$

en sorte que la loi de glissement prend la forme cinématique 


$$
f_{i, i+1}=k_{i, i+1}\left(u_{i+1}-u_{i}+\frac{1}{2}\left(t_{i+1}+t_{i}\right) v^{\prime}\right)
$$

On peut introduire cette loi dans les équations [1] et [3] précédentes, puis sommer les $N$ équations [3] - ce qui fait disparaitre les forces normales $p_{\mathrm{i}, i+1}$ - pour obtenir une formulation en purs déplacements, composée de cette somme et des $N$ équations [1] pour les $N+1$ inconnues $u_{\mathrm{i}}(x)$ et $v(x)$, pour autant que les forces normale et tangentielle aux fibres extrêmes du multicouche soient admises connues voire nulles.

\section{Forme faible linéaire}

Soit $\tilde{u}_{i}(x)$ et $\tilde{v}(x)$ deux fonctions poids ; pour la couche $i$, à partir de [1] et [3], avec la notation compactée

$$
f_{i}^{-}=f_{i, i+1}-f_{i-1, i} \quad f_{i}^{+}=f_{i, i+1}+f_{i-1, i} \quad p_{i}^{-}=p_{i, i+1}-p_{i-1, i}
$$

et sur la longueur $L$ de la poutre, on construit l'intégrale

$$
\int_{L}\left(E_{i} A_{i} u_{i}^{\prime \prime}+f_{i}^{-}+n_{i}\right) \tilde{u}_{i} d x+\int_{L}\left(-E_{i} I_{i} v^{\prime \prime \prime \prime}+\frac{1}{2} t_{i}\left(f_{i}^{+}\right)^{\prime}+p_{i}^{-}+q_{i}\right) \tilde{v} d x=0
$$

En intégrant par parties une fois les termes en $u_{i}^{\prime \prime}$ et $\left(f_{i}^{+}\right)^{\prime}$ et deux fois le terme en $v^{\prime \prime \prime \prime \prime}$, on obtient, avec $N_{i}=E_{i} A_{i} u_{i}^{\prime}, M_{i}=-E_{i} I_{i} v^{\prime \prime}$ et $V_{i}$ [2],

$$
\int_{L} N_{i} \tilde{u}_{i}^{\prime} d x+\int_{L} M_{i} \tilde{v}^{\prime \prime} d x-\int_{L} f_{i}^{-} \tilde{u}_{i} d x+\int_{L} \frac{1}{2} t_{i} f_{i}^{+} \tilde{v}^{\prime} d x-\int_{L} p_{i}^{-} \tilde{v} d x=\int_{L} n_{i} \tilde{u}_{i} d x+\int_{L} q_{i} \tilde{v} d x+T A L
$$

où l'on a regroupé dans la notation $T A L$ les termes aux limites

$$
T A L=N_{i}(L) \tilde{u}_{i}(L)-N_{i}(0) \tilde{u}_{i}(0)+V_{i}(L) \tilde{v}(L)-V_{i}(0) \tilde{v}(0)-M_{i}(L) \tilde{v}^{\prime}(L)+M_{i}(0) \tilde{v}^{\prime}(0)
$$

La forme faible est la somme sur les $N$ couches de l'équation [5]. Lors de cette sommation, le dernier terme du membre de gauche donne, avec les conditions aux limites $p_{0,1}=p_{N, N+1}=0$,

$$
\sum_{1}^{N} \int_{L} p_{i}^{-} \tilde{v} d x=0
$$

et les deuxième et troisième termes peuvent être regroupés comme suit, avec les conditions aux limites $f_{0,1}=f_{N, N+1}=0$, 


$$
\sum_{1}^{N}\left(-\int_{L} f_{i}^{-} \tilde{u}_{i} d x+\int_{L} \frac{1}{2} t_{i} f_{i}^{+} \tilde{v}^{\prime} d x\right)=\sum_{1}^{N-1} \int_{L} f_{i, i+1} \tilde{g}_{i, i+1} d x
$$

où apparaît, avec [4], la fonction poids de glissement

$$
\tilde{g}_{i, i+1}=\tilde{u}_{i+1}-\tilde{u}_{i}+\frac{1}{2} t_{i+1} \tilde{v}^{\prime}+\frac{1}{2} t_{i} \tilde{v}^{\prime}
$$

En tenant compte de ce qui précède, la forme faible peut s'écrire

$\sum_{N}\left(\int_{L} N_{i} \tilde{u}_{i}^{\prime} d x+\int_{L} M_{i} \tilde{v}^{\prime \prime} d x\right)+\sum_{N-1} \int_{L} f_{i, i+1} \tilde{g}_{i, i+1} d x=\sum_{N}\left(\int_{L} n_{i} \tilde{u}_{i} d x+\int_{L} q_{i} \tilde{v} d x\right)+\sum_{N} T A L$

Elle s'accompagne des conditions aux limites essentielles sur $u_{i}, v$ et $v^{\prime}$, et prend immédiatement ce caractère physique intuitif propre au principe des travaux virtuels lorsqu'on interprète les fonctions poids comme des déplacements virtuels.

REMARQUE. - Bien qu'en présence de glissement des interfaces les résultantes des efforts intérieurs n'aient plus guère de sens, on peut, par exemple, les écrire par rapport à l'axe moyen de la première couche

$$
N_{\mathrm{r}}=\sum_{1}^{N} N_{i} \quad V_{\mathrm{r}}=\sum_{1}^{N} V_{i} \quad M_{\mathrm{r}}=\sum_{1}^{N}\left(M_{i}+N_{i} e_{i}\right)
$$

\section{Forme matricielle linéaire}

Cette forme s'obtient en combinant le choix d'une approximation polynomiale des champs cinématiques à celui des déplacements inconnus nodaux. On appelle $\mathrm{A}$ et B les extrémités d'un tronçon de poutre de longueur $L=2 a$, au centre $\mathrm{C}$ duquel on place l'origine des axes. Le déplacement axial $u_{i}(x)$ de chaque couche $i$ est choisi quadratique, le second degré étant exprimé par un terme hiérarchique

$$
u_{i}(x)=u_{i \mathrm{~A}} N_{\mathrm{A}}(x)+u_{i \mathrm{~B}} N_{\mathrm{B}}(x)+\Delta u_{i} N_{\mathrm{C}}(x)
$$

où $u_{i \mathrm{~A}}$ et $u_{i \mathrm{~B}}$ sont les déplacements axiaux en $\mathrm{A}$ et $\mathrm{B}, \Delta u_{i}$ le déplacement hiérarchique en $\mathrm{C}$, et $N_{\mathrm{A}}(x)=(1-x / a) / 2, N_{\mathrm{B}}(x)=(1+x / a) / 2$ et $N_{\mathrm{C}}(x)=1-(x / a)^{2}$ les fonctions d'interpolation. Le déplacement transversal $v(x)$ cubique, exprimé avec les fonctions $C^{1}$ de Hermite $M_{\mathrm{A}}(x), L_{\mathrm{A}}(x), M_{\mathrm{B}}(x)$ et $L_{\mathrm{B}}(x)$ (Batoz et al., 1990), est donné par

$$
v(x)=v_{\mathrm{A}} M_{\mathrm{A}}(x)+v_{\mathrm{A}}^{\prime} L_{\mathrm{A}}(x)+v_{\mathrm{B}} M_{\mathrm{B}}(x)+v_{\mathrm{B}}^{\prime} L_{\mathrm{B}}(x)
$$

où $v_{\mathrm{A}}, v_{\mathrm{B}}, v_{\mathrm{A}}^{\prime}$ et $v_{\mathrm{B}}^{\prime}$ sont les déplacements transversaux et les rotations (pentes) en $\mathrm{A}$ et $\mathrm{B}$. 
Les composantes horizontales $u_{i \mathrm{~A}}$ et $u_{\mathrm{B}}$ des vecteurs déplacements des nœuds, pour $i=2$ à $N$, sont malaisées à manipuler lors de rotations des axes ou de grands déplacements. On leur substitue avantageusement les scalaires glissements $g_{i, i+1 \mathrm{~A}}$ et $g_{i, i+1 \mathrm{~B}}, i=1, N-1$. Le vecteur déplacement de l'élément fini est ainsi (avant condensation des degrés de liberté en $\Delta$ )

$$
\begin{aligned}
& \mathbf{d}^{\mathrm{T}}=\left\{u_{1 \mathrm{~A}} v_{\mathrm{A}} v_{\mathrm{A}}^{\prime} g_{12 \mathrm{~A}} g_{23 \mathrm{~A}} \ldots g_{N-1, N \mathrm{~A}}\left|u_{1 \mathrm{~B}} v_{\mathrm{B}} v_{\mathrm{B}}^{\prime} g_{12 \mathrm{~B}} g_{23 \mathrm{~B}} \ldots g_{N-1, N \mathrm{~B}}\right|\right. \\
&\left.\Delta u_{1} \Delta g_{12} \Delta g_{23} \ldots \Delta g_{N-1, N}\right\}
\end{aligned}
$$

De [4], on déduit aisément, pour $i=2, N$, où $e_{i}$ est l'ordonnée de l'axe de la couche $i$ (figure 1),

$$
u_{i}(x)=u_{1}(x)+\sum_{j=1}^{i-1} g_{j, j+1}(x)-e_{i} v^{\prime}(x)
$$

Le champ $g_{i, i+1}(x)$ est interpolé quadratiquement à l'image de $u_{\mathrm{i}}(x)$ [7]

$$
g_{i, i+1}(x)=g_{i, i+1 \mathrm{~A}} N_{\mathrm{A}}(x)+g_{i, i+1 \mathrm{~B}} N_{\mathrm{B}}(x)+\Delta g_{i, i+1} N_{\mathrm{C}}(x)
$$

Le champ $v^{\prime}(x)$, dérivée de $v(x)$, est également quadratique et pourrait donc s'écrire $v^{\prime}(x)=v_{\mathrm{A}}^{\prime} N_{\mathrm{A}}(x)+v_{\mathrm{B}}^{\prime} N_{\mathrm{B}}(x)+\Delta v^{\prime} N_{\mathrm{C}}(x)$, soit sous la même forme que [7]; on en déduit, entre les degrés de liberté hiérarchiques, la relation

$$
\Delta u_{i}=\Delta u_{1}+\sum_{j=1}^{i-1} \Delta g_{j, j+1}-e_{i} \Delta v^{\prime}
$$

avec

$$
\Delta v^{\prime}=v^{\prime}(0)-v_{\text {linéaire }}^{\prime}(0)=\frac{3}{4}\left(\frac{1}{a}\left(v_{\mathrm{B}}-v_{\mathrm{A}}\right)-\left(v_{\mathrm{B}}^{\prime}+v_{\mathrm{A}}^{\prime}\right)\right)
$$

A l'aide des interpolations précédentes et du vecteur $\mathbf{d}$, on peut, via la forme faible [6], calculer la matrice de rigidité et le vecteur force de l'élément fini (les fonctions poids sont interpolées identiquement - méthode de Galerkin). On condense ensuite, avant assemblage, les degrés de liberté hiérarchiques pour ne conserver d'inconnues qu'aux deux nœuds $\mathrm{A}$ et $\mathrm{B}$, en nombre $2(N+2)$ (ce qui correspond à la première ligne de [9]). Rappelons que les modes hiérarchiques axiaux permettent d'éviter les verrouillages (tant en linéaire qu'en non linéaire). 


\section{Extensions}

\subsection{Elément courbe}

Soit $v_{0}(x)$ l'équation de l'axe d'un élément courbe, supposé à faible courbure ( shallow $\left.\Rightarrow v^{\prime}{ }_{0}(x) \leq \sim 0,1 \mathrm{rad}\right)$, décrite par rapport à la corde de l'élément et en coordonnées cartésiennes (figure 4$) ; v_{0}(x)$ est généralement choisie cubique comme $v(x)[8]$ (avec $v_{0 \mathrm{~A}}$ et $v_{0 \mathrm{~B}}$ nuls)

$$
v_{0}(x)=v_{0 \mathrm{~A}}^{\prime} L_{\mathrm{A}}(x)+v_{0 \mathrm{~B}}^{\prime} L_{\mathrm{B}}(x)
$$

L'introduction de $v_{0}$ se marque par l'apparition du terme $\varepsilon_{M}$, dit de Marguerre, dans la mesure de la dilatation axiale

$$
\varepsilon_{M}=v_{0}^{\prime} v^{\prime}
$$

Pour éviter un verrouillage sévère, il convient de moyenner ce terme sur l'élément (assumed strain method) :

$$
\varepsilon_{M} \mapsto \bar{\varepsilon}_{M}=\frac{1}{2 a} \int_{-a}^{a} v_{0}^{\prime} v^{\prime} d x
$$

Ce terme ne touche que le déplacement transversal commun à toutes les couches ; ses apports à la matrice de rigidité (somme sur les $N$ couches) sont donc les mêmes que pour une poutre monosection et ont déjà été étudiés ailleurs (par exemple (Frey et al., 1992)).

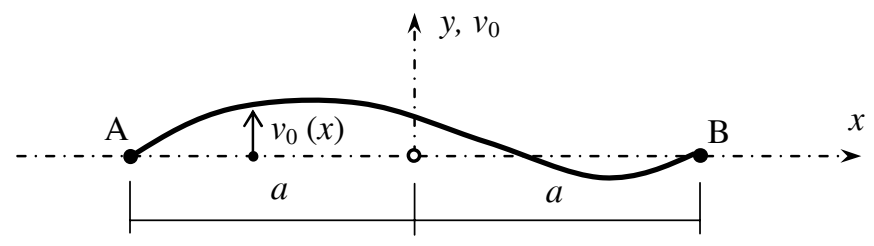

Figure 4. Elément courbe par une déformée initiale surbaissée

\subsection{Comportement inélastique}

Si les matériaux ou les surfaces de glissement ont un comportement inélastique, par exemple élasto-plastique, on ne peut plus travailler en efforts intérieurs dans [6] ; il faut intégrer sur la surface $A_{i}=b_{i} t_{i}$ des diverses couches et remplacer les deux premiers termes de [6] par 


$$
\sum_{N}\left(\int_{L} N_{i} \tilde{u}_{i}^{\prime} d x+\int_{L} M_{i} \tilde{v}^{\prime \prime} d x\right) \mapsto \sum_{N} \int_{L} \int_{A_{i}} \sigma_{i} \tilde{\varepsilon}_{i} d A d x
$$

avec $\sigma_{i}=E_{t i} \varepsilon_{i}$ et, dans le troisième terme de [6], $f_{i, i+1}=k_{t i, i+1} g_{i, i+1}$, où $E_{t i}$ et $k_{t i, i+1}$ sont les modules tangents aux lois de comportement de la couche $i$ et de l'interface $i, i+1$. Dans la couche $i(i=2, N)$, on appelle $y_{i}$ l'ordonnée locale $\left(-t_{i} / 2 \leq y_{i} \leq t_{i} / 2\right)$ et $\bar{u}_{i}\left(x, y_{i}\right)$ le déplacement longitudinal; on a, avec [10] (poutre de Bernoulli),

$$
\bar{u}_{i}\left(x, y_{i}\right)=u_{i}(x)-y_{i} v^{\prime}(x)=u_{1}(x)+\sum_{j=1}^{i-1} g_{j, j+1}(x)-\left(e_{i}+y_{i}\right) v^{\prime}(x)
$$

avec, pour la couche $1, \bar{u}_{1}\left(x, y_{1}\right)=u_{1}(x)-y_{1} v^{\prime}(x)$ simplement. On peut maintenant calculer

$$
\varepsilon_{i}\left(x, y_{i}\right)=\bar{u}_{i}^{\prime}\left(x, y_{i}\right)
$$

et, exprimant $\tilde{\varepsilon}_{i}$ semblablement, écrire ces termes en fonction des incréments des déplacements et, enfin, évaluer les termes de la matrice de rigidité tangente (intégration numérique), le tout en vue d'une résolution incrémentielle (NewtonRaphson).

\subsection{Grands déplacements}

Les grands déplacements sont traités, au niveau de l'élément fini, par la formulation corotationnelle (par exemple (Crisfield, 1991; Belytschko et al., 2000)) ; en axes actualisés, en supposant les déformations constamment petites, on peut adopter, comme mesure de la cinématique non linéaire, la déformation de von Karman

$$
\varepsilon_{v K}=\frac{1}{2} v^{2}
$$

Comme pour Marguerre, il convient d'en prendre la moyenne pour combattre le verrouillage :

$$
\varepsilon_{v K} \mapsto \bar{\varepsilon}_{v K}=\frac{1}{2 a} \frac{1}{2} \int_{-a}^{a} v^{\prime 2} d x
$$

A nouveau, ce terme ne touche que le déplacement transversal commun à toutes les couches et contribue à la matrice de rigidité tangente (linéarisation) de la même manière qu'une poutre monosection (somme sur les $N$ couches). On trouvera ces contributions ailleurs (par exemple (Frey et al., 1992)). La résolution est 
incrémentielle de type Newton-Raphson; la matrice des contraintes initiales permet le calcul d'instabilité linéarisée (problème aux valeurs propres conduisant à la charge critique d'Euler).

\section{Tests numériques}

Pour valider et illustrer le potentiel de l'élément fini développé, on présente cinq tests, qui couvrent l'élasticité linéaire, l'instabilité linéaire et les non-linéarités géométrique et matérielle. Les trois premiers sont comparés à des solutions analytiques, les deux derniers à des essais.

\subsection{Poutre console}

Une console est formée de deux couches superposées identiques à section rectangulaire $t \cdot b$ (figure 5). La rigidité $k$ de l'interface est admise constante sur toute la longueur $L$ de la poutre. Le rapport $h / L$ de la hauteur totale $h$ de la poutre à sa portée est égal à $1 / 10$. En régime matériellement et géométriquement linéaire et pour une charge répartie uniforme $q$, la solution analytique de la force d'interface $f$ (x) livrée par (Girhammar et al., 1993) est donnée par

$$
f(x)=\frac{3}{2} \frac{q L}{b h}\left(1-\frac{x}{L}+\frac{\operatorname{sh} \alpha x}{\alpha L \operatorname{ch} \alpha L}-\frac{\operatorname{ch} \alpha(L-x)}{\operatorname{ch} \alpha L}\right)
$$

où $\alpha^{2}=8 k / E A\left[1 / \mathrm{mm}^{2}\right]$ et où $k$ est le module de glissement.

La figure 6 présente la distribution de la force d'interface pour deux valeurs du module de glissement $k=0,1 E$ et $0,001 E$. Dans le premier cas, le module est suffisamment fort pour provoquer une brusque variation de la force à proximité de l'encastrement; à l'extrémité libre, le glissement est encore possible et la force y vaut environ $2 \%$ de sa valeur maximale. Dans le second cas, la variation est plus douce.

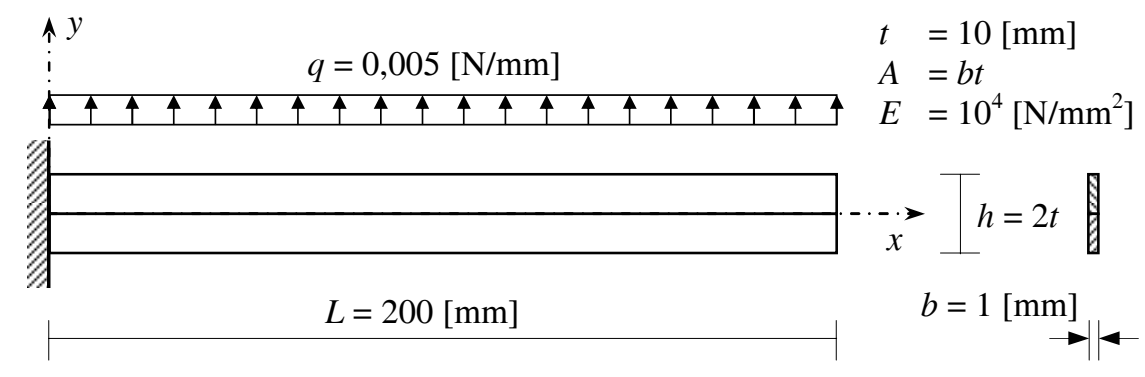

Figure 5. Poutre console à deux couches chargée uniformément 


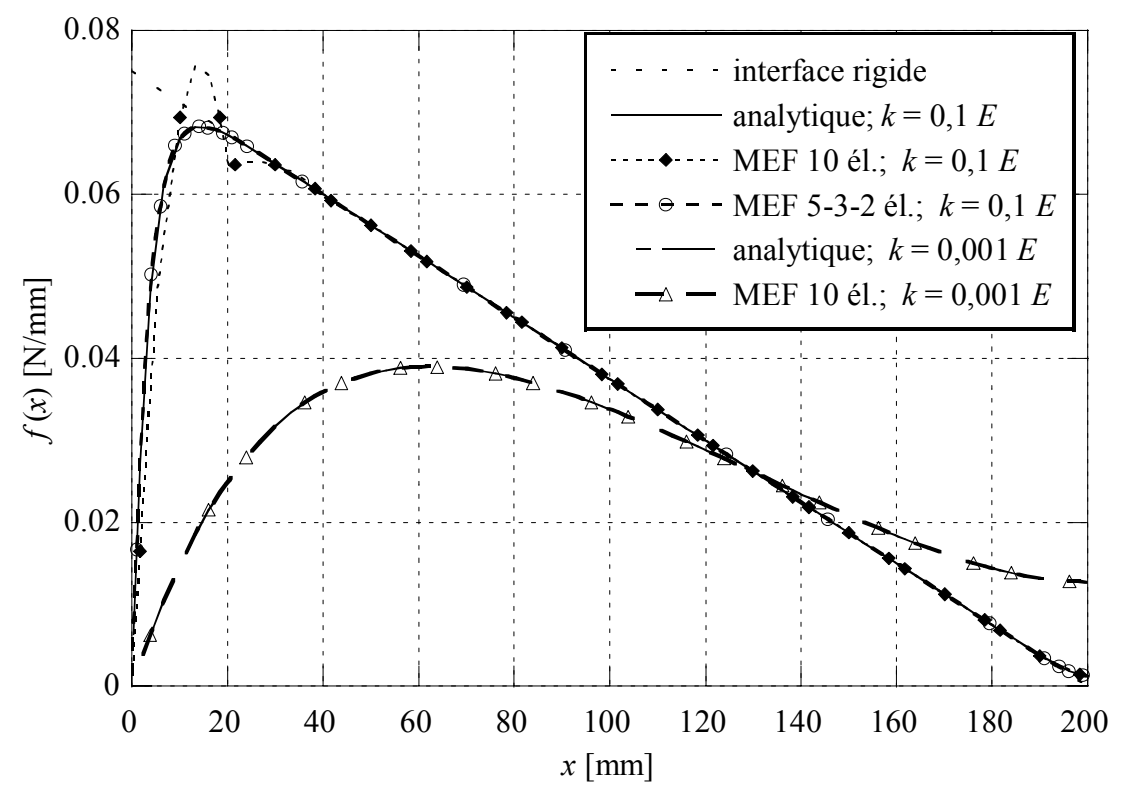

Figure 6. Distribution de la force tangentielle d'interface

Le premier cas est simulé à l'aide de deux réseaux d'éléments finis. L'un est constitué de dix éléments réguliers de $20 \mathrm{~mm}$ de longueur, l'autre comprend le même nombre d'éléments mais répartis en fonction de la variation de la force d'interface : 5 éléments sur les 25 premiers millimètres, 3 sur les 165 suivants et 2 sur les 10 derniers. A cause de la variation exponentielle du glissement, le réseau régulier présente une solution imprécise principalement dans la zone d'encastrement (erreur de l'ordre de $25 \%$ ). Le second réseau, mieux adapté (la force d'interface, étant directement liée au champ de déplacements, a un caractère superconvergent), approche la solution à environ $5 \%$.

Le second cas est simulé uniquement avec le réseau régulier. La variation de la force d'interface y étant moindre, l'erreur est fortement réduite : de l'ordre de 1,3\% à l'encastrement à moins de $0,1 \%$ au bord libre. Pour les deux cas, la flèche à l'extrémité libre est très peu affectée par les erreurs de précision sur la distribution de la force d'interface.

\subsection{Flambement d'une poutre en $\mathrm{T}$}

Ce test est tiré de (Kamiya, 1987). Il s'agit d'une poutre simplement appuyée dont la section en $\mathrm{T}$ est formée d'une âme en bois et d'une semelle en béton (les dimensions sont données à la figure 7). La connexion entre les deux matériaux est 
assurée par des clous : son module de glissement est $k=49\left[\mathrm{~N} / \mathrm{mm}^{2}\right]$. Les modules d'élasticité respectifs du bois et du béton sont $E_{1}=7840\left[\mathrm{~N} / \mathrm{mm}^{2}\right]$ et $E_{2}=4900$ $\left[\mathrm{N} / \mathrm{mm}^{2}\right]$. Avec un réseau de trente éléments finis réguliers, on obtient une charge critique linéarisée $P_{\mathrm{cr}}^{\mathrm{MEF}}=58,033[\mathrm{kN}]$ qui est identique à celle que proposent (Kamiya, 1987 ; Girhammar et al., 1993).

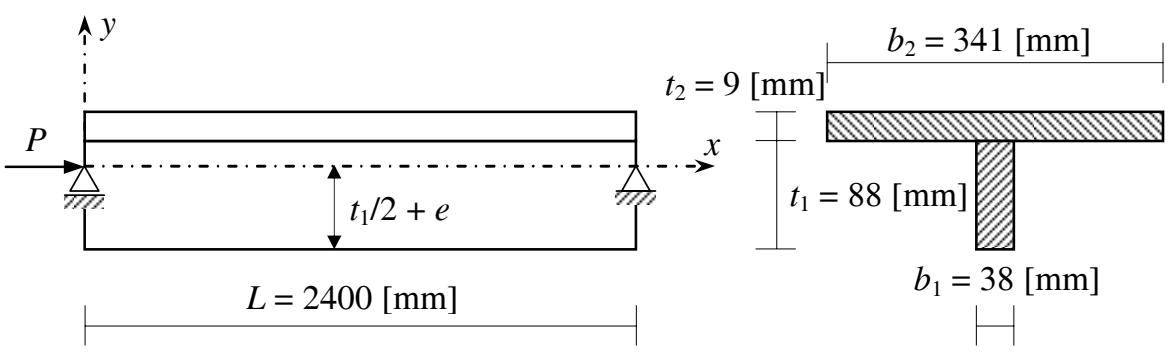

Figure 7. Schéma statique et géométrie de la poutre

Comme l'a démontré (Kamiya, 1987), ce type de poutre (simplement appuyée, section composite en $\mathrm{T}$, loi de glissement linéaire à l'interface) n'a pas, en dessous de la charge critique, de configuration d'équilibre transversalement non déformée, comme c'est le cas d'une colonne monolithique parfaite. Cet auteur a introduit la notion de centre de compression qui définit la position de la charge axiale pour laquelle l'amplitude de la déformée transversale est minimale. La position de ce centre dépend de la rigidité de l'interface; si on choisit comme point de référence le milieu de l'âme, le centre de compression se déplace asymptotiquement de ce point vers le centre géométrique de la section quand la rigidité de l'interface croît de zéro à l'infini. Pour ce test, il se situe à $e \cong 13,25[\mathrm{~mm}]$. Si la charge $P$ est appliquée d'un côté ou de l'autre de ce centre, le comportement de la poutre change comme le tableau 1 le montre à travers le signe et l'amplitude de la flèche transversale à mi-portée.

\begin{tabular}{|c|c|c|c|}
\hline & $e=15,00[\mathrm{~mm}]$ & $e=13,25[\mathrm{~mm}]$ & $e=11,50[\mathrm{~mm}]$ \\
\hline linéaire, MEF & $-1,1356$ & 0,3308 & 1,7972 \\
\hline non linéaire, MEF & $-4,4737$ & 0,3822 & 5,2384 \\
\hline $\begin{array}{c}\text { second ordre, analytique } \\
\text { (Kamiya, 1987) }\end{array}$ & $-4,4805$ & 0,3823 & 5,2451 \\
\hline
\end{tabular}

Tableau 1. Flèches au milieu de la poutre, pour $P=40[\mathrm{kN}]$ 
Pour une charge $P=40[\mathrm{kN}]$, ce changement, déjà apparent dans l'analyse linéaire, est amplifié d'un facteur de l'ordre de 2,5 dans l'analyse numérique non linéaire, pour laquelle on observe une erreur inférieure à $0,2 \%$ par rapport à la solution du second ordre de (Kamiya, 1987). La figure 8 met en évidence les configurations déformées associées à la position $e$ de la charge.

\subsection{Flexion pure d'une poutre console bilame}

Pour tester le comportement du modèle multicouche en grands déplacements, on fléchit une console formée de deux couches en quart de cercle, avec glissement libre entre les couches. La poutre a une longueur $L=100[\mathrm{~mm}]$; les deux couches ont une largeur commune $b_{1}=b_{2}=1[\mathrm{~mm}]$ et des hauteurs $t_{1}=3[\mathrm{~mm}]$ et $t_{2}=1[\mathrm{~mm}]$ (figure 9).

A l'extrémité libre de la première couche est appliqué un moment $M_{1}$ de manière à déformer cette couche en un quart de cercle. Le rayon $R_{1}$ de l'axe de la couche et le moment $M_{1}$ sont donnés par $1 / R_{1}=\pi / 2 L=M_{1} / E_{1} I_{1}$.

La seconde couche est astreinte à former un cercle, concentrique au premier, de rayon $R_{2}=R_{1}-\left(t_{1}+t_{2}\right) / 2$, attendu que les deux couches glissent l'une sur l'autre sans déplacement relatif ni réaction transverses. Le moment $M_{2}$ qu'il faut appliquer à l'extrémité libre de cette couche est issu de la relation $1 / R_{2}=M_{2} / E_{2} I_{2}$. Le glissement en fonction de $x$ s'écrit

$$
g(x)=\frac{x}{R_{2}}\left(R_{2}+\frac{t_{2}}{2}\right)-\frac{x}{R_{1}}\left(R_{1}-\frac{t_{1}}{2}\right)=\frac{x}{2}\left(\frac{t_{1}}{R_{1}}+\frac{t_{2}}{R_{2}}\right)
$$

Partant de ce modèle, la poutre est simulée par un réseau de 10 éléments finis de deux couches (avec le module de glissement de l'interface $k=0$ ). L'élément fini ne possédant qu'un seul degré de liberté en rotation, commun à toutes les couches, il n'a également qu'une seule condition naturelle associée : le moment appliqué $M$ doit être la somme deux moments $\mathrm{M}_{1}$ et $\mathrm{M}_{2}$ définis ci-avant. Les propriétés géométriques et mécaniques sont données à la figure 9. Le tableau 2 compare les principaux résultats. Bien qu'il s'agisse d'un maillage relativement grossier, la précision obtenue est excellente. Il faut néanmoins remarquer que, s'agissant des moments dans les deux couches, la répartition rendue par les éléménts n'est pas celle prévue par le modèle analytique. Les éléments finis redistribuent les moments proportionnellement à la rigidité des couches, en fait comme si l'axe moyen de la seconde couche était superposé à celui de la première. Cela provient de l'existence d'un seul champ de déplacement transversal et d'un unique degré de liberté en rotaton, tous deux communs aux diverses couches. 
$e=15,0[\mathrm{~mm}]$

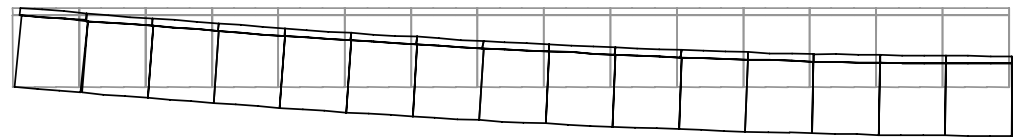

$P=56,0$

$[\mathrm{kN}]$

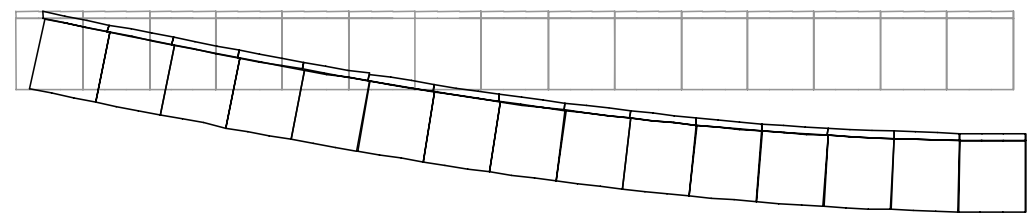

$P=57,5$

$e=13,25[\mathrm{~mm}]$
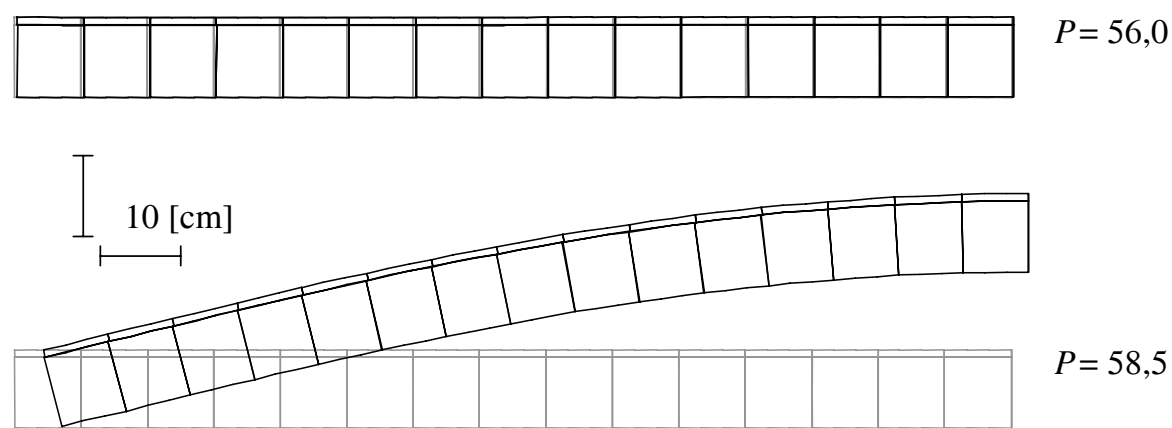

$e=11,5[\mathrm{~mm}]$

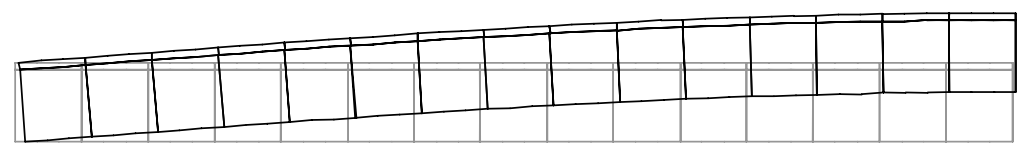

$P=56,0$

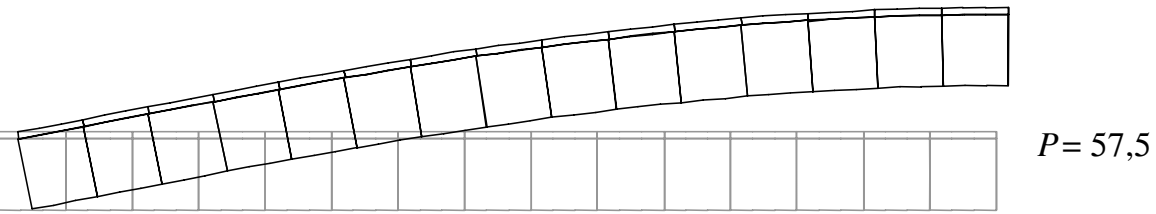

Figure 8. Configurations déformées d'une demi-poutre en fonction de la position de la charge (à l'échelle) 


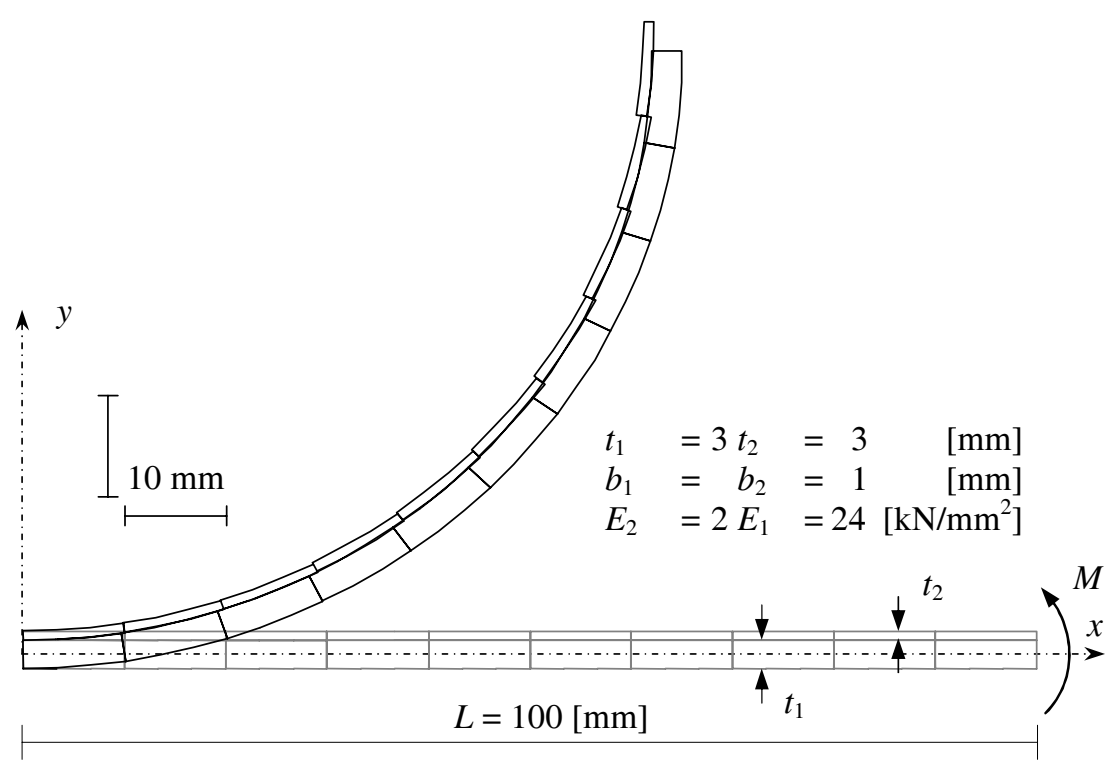

Figure 9. Poutre console bilame en grands déplacements (à l'échelle)

\begin{tabular}{|c|c|c|c|}
\cline { 2 - 4 } \multicolumn{1}{c|}{} & Analytique & MEF 10 éléments & erreur \\
\hline$v(x=L)[\mathrm{mm}]$ & $R_{1}=63,662$ & 63,743 & $0,1 \%$ \\
\hline$u(x=L)[\mathrm{mm}]$ & $R_{1}-L=-36,338$ & $-36,480$ & $0,4 \%$ \\
\hline$g(x=L)[\mathrm{mm}]$ & 3,167 & 3,149 & $0,6 \%$ \\
\hline$M[\mathrm{Nmm}]$ & $M_{1}=-424,115$ & $M_{1}=-425,064$ & $0,2 \%$ \\
& $M_{2}=-32,435$ & $M_{2}=-31,486$ & $2,9 \%$ \\
\hline $\max |N(x)|[\mathrm{N}]$ & $N_{1}=N_{2}=0$ & $0,68210^{-12}$ & - \\
\hline
\end{tabular}

Tableau 2. Flexion pure en grands déplacements de la console bilame

\subsection{Poutre continue mixte à deux travées égales}

Yam et al., (1972) ont étudié le comportement d'une poutre continue mixte acierbéton à deux travées égales sollicitées par une charge $P$ concentrée en leur milieu. La connexion entre les deux matériaux est assurée par des goujons uniformément espacés. La géométrie et le schéma statique sont dessinés à la figure 10. Comme la 
position des armatures longitudinales par rapport au sommet du béton n'est pas donnée de manière explicite par (Yam et al., 1972), elle a été estimée à $20 \%$ de l'épaisseur de béton. Les matériaux et la connexion par les goujons suivent des lois de comportement non linéaires, de type uniaxial, que fournit la référence et dont les paramètres sont résumés dans le tableau 3. Il faut encore préciser qu'en compression, le béton se rompt dès que la déformation atteint $\varepsilon_{\mathrm{c}}$. De plus, en l'absence de donnée sûre, la loi utilisée pour simuler le comportement des goujons ne comporte pas de limite de glissement de rupture.

Pour l'analyse par éléments finis, un réseau de 20 éléments par travée a été choisi. Le profilé métallique est simulé par trois couches d'acier correspondant aux deux semelles et à l'âme. L'aire des armatures longitudinales est répartie uniformément sur la largeur du tablier et forme également une couche. Le glissement entre les couches d'acier ainsi qu'entre la couche d'armatures et les deux couches de béton est empêché par un module de glissement très grand $\left(k=10^{8}\left[\mathrm{~N} / \mathrm{mm}^{2}\right]\right)$.

La charge de ruine de l'essai est de $P_{\mathrm{u}}^{\text {essai }}=150,4[\mathrm{kN}]$. La ruine a lieu, selon (Yam et al., 1972), par rupture de l'interface. Les auteurs notent aussi que, pour l'une des deux travées, le béton présente quelques prémices d'éclatements à proximité de la charge, un peu avant la ruine à $P=149,4[\mathrm{kN}]$, annonçant une ruine par écrasement du béton par flexion. Le faible écart entre le niveau de ces deux charges ne permet pas d'affirmer de manière certaine que la ruine est due plutôt à la première cause qu'à la seconde et donc que la première doit obligatoirement provoquer la ruine. Dans l'analyse par éléments finis, en l'absence de critère limitant le glissement, la ruine survient par écrasement du béton sous les charges pour $P_{\mathrm{u}}^{\mathrm{MEF}}=142,9[\mathrm{kN}]$, c'est-à-dire à $95 \%$ de la charge de l'essai. Ce résultat est très satisfaisant. L'écart trouvé peut s'expliquer par exemple par l'absence de résistance à la traction du béton, car au voisinage des charges, des tensions apparaissent très tôt à la base du tablier à cause du glissement de l'interface acier/béton.

Les figures 11 et 12 comparent respectivement les glissements obtenus à l'interface acier/béton et la déformée pour $P=121,5[\mathrm{kN}]$. L'écart maximal entre l'essai et le calcul est de l'ordre de $20 \%$ dans les deux cas. Les résultats de l'essai présentent une asymétrie assez prononcée. Ce défaut de symétrie n'est pas commenté par (Yam et al., 1972). Néanmoins, il peut être intéressant d'évaluer l'effet d'une dissymétrie des caractéristiques de la connexion acier/béton. En augmentant de $10 \%$ les propriétés de l'interface de la travée de gauche et en réduisant d'autant celles de la travée de droite, on observe une amélioration des résultats; l'asymétrie des conditions d'essai (par exemple au niveau des connexions) est une cause probable de l'asymétrie des grandeurs mesurées. 


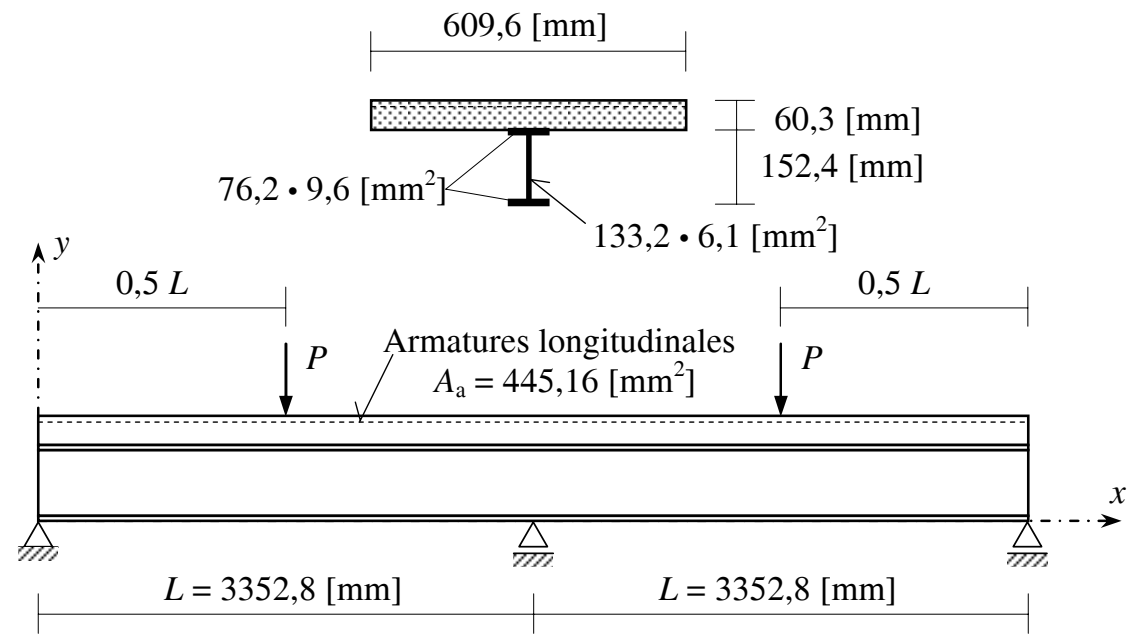

Figure 10. Schéma statique et géométrie de la poutre continue

\begin{tabular}{|l|c|r|r|r|}
\hline \multicolumn{1}{|c|}{ Composant } & Loi constitutive & $\begin{array}{r}\text { Module } \\
\text { initial } E_{0} \\
{\left[\mathrm{~N} / \mathrm{mm}^{2}\right]}\end{array}$ & $\begin{array}{c}\text { Module } \\
\text { d'écrouissage } \\
E_{\mathrm{t}} \\
{\left[\mathrm{N} / \mathrm{mm}^{2}\right]}\end{array}$ & $\begin{array}{c}\text { Résistance } f_{\mathrm{y}} \\
\text { ou } \sigma_{\mathrm{u}} \\
{\left[\mathrm{N} / \mathrm{mm}^{2}\right]}\end{array}$ \\
\hline Profilé en acier & bilinéaire & 207000,0 & 1034,5 & 301,80 \\
\hline Acier d'armature & élasto-plast. & 207000,0 & 0,0 & 321,15 \\
\hline Béton : compression & élasto-plast. & 16270,8 & 0,0 & 47,58 \\
traction & sans résistance & & -- & 5,83 \\
\hline Goujons $\quad f(g)=$ & $\sigma_{\mathrm{u}}\left(1-\mathrm{e}^{-E_{0} g / \sigma_{\mathrm{u}}}\right)$ & 27,53 & & \\
\hline
\end{tabular}

Tableau 3. Lois constitutives

\subsection{Poutre simple formée de planches en bois clouées}

Cette poutre est constituée de cinq planches $(5 \cdot 300 \cdot 7,8 \cdot 2,7[\mathrm{~cm}])$ superposées et connectées les unes aux autres à l'aide de clous de diamètre $2,9 \mathrm{~mm}$ répartis régulièrement tous les $80 \mathrm{~mm}$. La longueur des clous permet de traverser trois planches et leur disposition est telle que les trois interfaces inférieures sont traversées par deux fois plus de clous que l'interface supérieure. La figure 13 montre le schéma statique et la géométrie de l'essai (Hübner, 2000). 


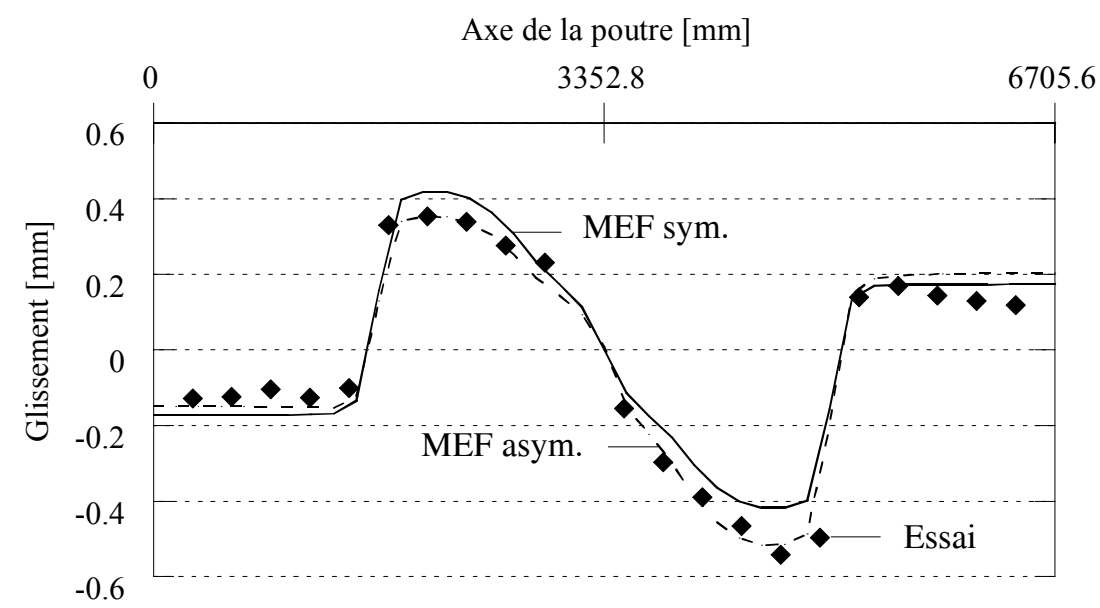

Figure 11. Comparaison des glissements de l'interface pour $P=121,5[\mathrm{kN}](M E F$ sym.: propriétés des connexions identiques; $M E F$ asym.: propriétés des connexions distinctes)

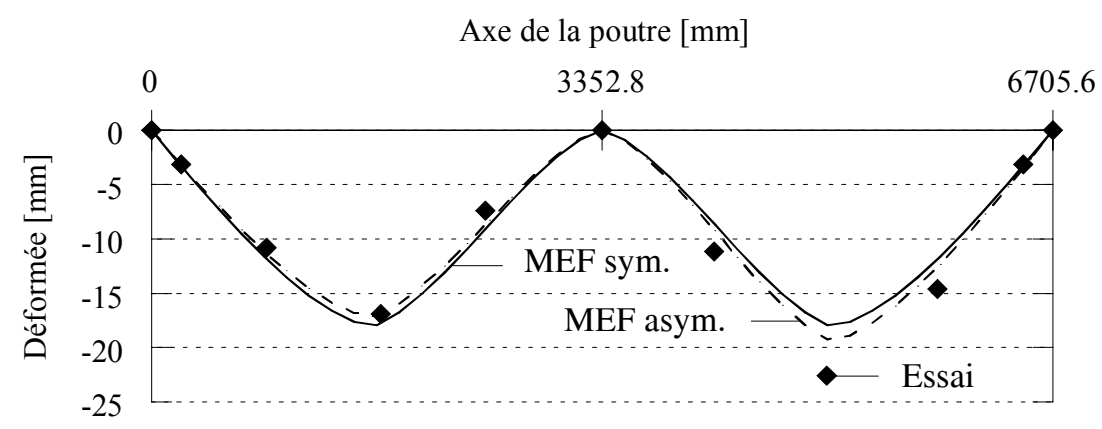

Figure 12. Comparaison des déformées pour $P=121,5[\mathrm{kN}]$

Pour la modélisation, la loi constitutive du bois est admise élastique linéaire $(E=$ $1420\left[\mathrm{kN} / \mathrm{cm}^{2}\right]$ ). Le comportement des interfaces (figure 14) est tiré d'essais effectués sur des éprouvettes à deux interfaces, de bois de mêmes essence et qualité, et de clous de diamètre 3,8 mm (Pirazzi, 2005). Une loi basée sur (Richard, 1961) s'adapte bien à l'allure des courbes d'essais dans la zone utile pour l'analyse avant rupture. On a pris une loi moyenne sur cinq essais puis adapté ses paramètres en fonction de la répartition et du diamètre effectifs des clous pour chaque interface (tableau 4). Le maillage utilisé tient compte de la symétrie et comprend 13 éléments. L'analyse est faite en grands déplacements jusqu'à proximité de la charge de ruine $\left(P_{\mathrm{u}}=5,4[\mathrm{kN}]\right)$. 

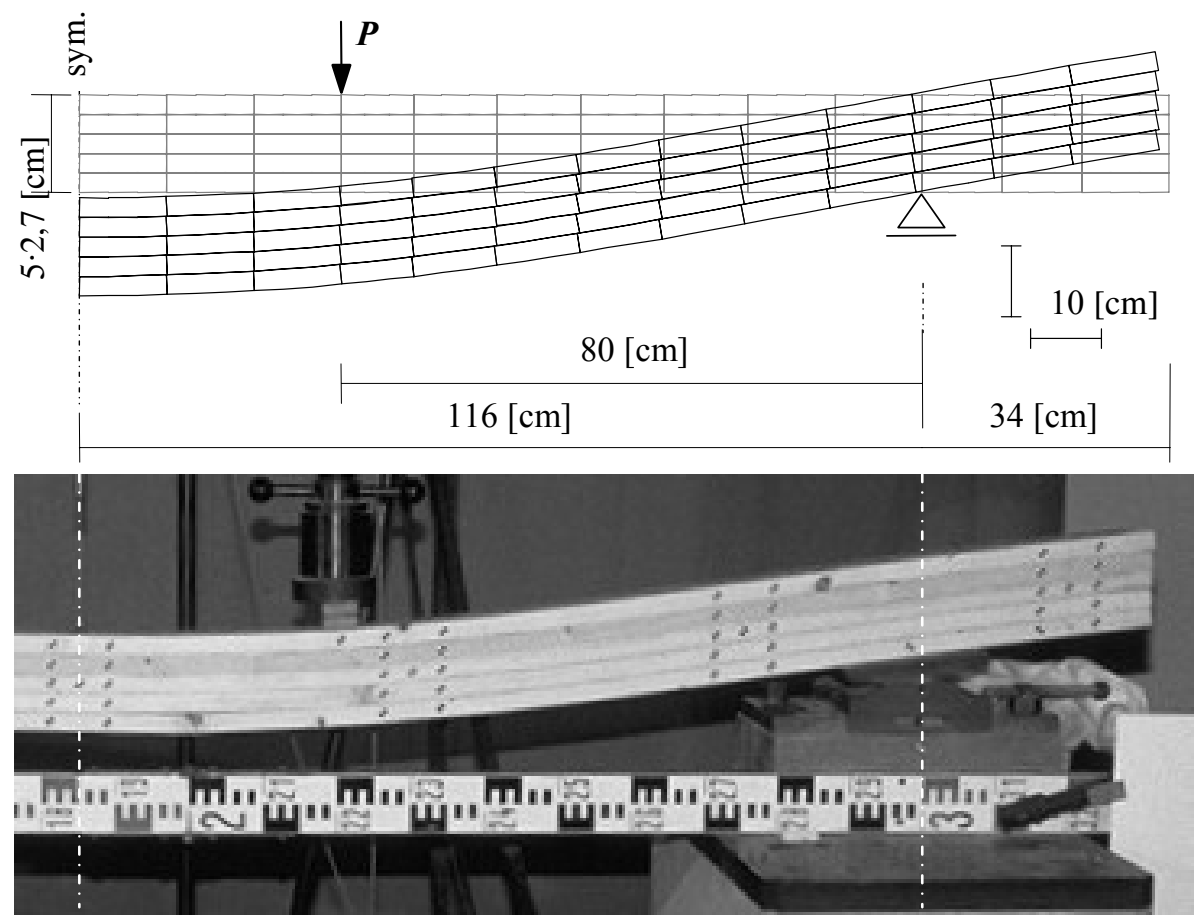

Figure 13. Schéma statique et dimensions de la poutre (demi-poutre par symétrie) ; comparaison des configurations déformées (à l'échelle)

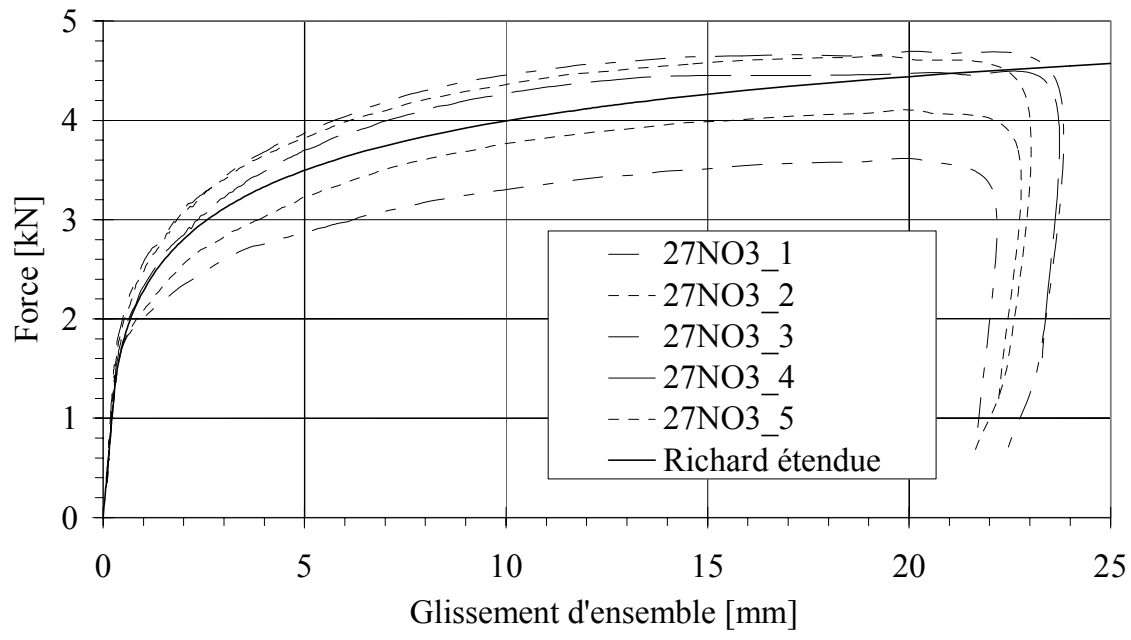

Figure 14. Courbes d'essais et loi de Richard moyenne 
Le tableau 5 compare les flèches en travée et les glissements aux extrémités de la poutre pour les niveaux de charge $P=2,5[\mathrm{kN}]$ et $P=5,3[\mathrm{kN}]$. On observe une très bonne concordance; le modèle représente bien le comportement de la poutre, en particulier le changement de courbure à proximité et au-delà de l'appui (figure 13).

\begin{tabular}{|c|c|c|c|c|}
\hline$\sigma=\sigma_{\mathrm{y}}+\frac{k g-\sigma_{\mathrm{y}}}{\left[1+\left(\frac{k g-\sigma_{\mathrm{y}}}{\sigma_{\mathrm{u}}-\sigma_{\mathrm{y}}}\right)^{r}\right]^{1 / r}}$ & $\begin{array}{c}k \\
{\left[\mathrm{kN} / \mathrm{cm}^{2}\right]}\end{array}$ & $\begin{array}{c}\sigma_{\mathrm{y}} \\
{\left[\mathrm{kN} / \mathrm{cm}^{2}\right]}\end{array}$ & $\begin{array}{c}\sigma_{\mathrm{u}} \\
{\left[\mathrm{kN} / \mathrm{cm}^{2}\right]}\end{array}$ & $r$ \\
\hline Interface & 0,2277 & 0,003391 & 0,01543 & 0,494 \\
\hline Interfaces & 0,4559 & 0,006782 & 0,03087 & 0.494 \\
\hline
\end{tabular}

Tableau 4. Lois de Richard: adaptation des paramètres pour les interfaces

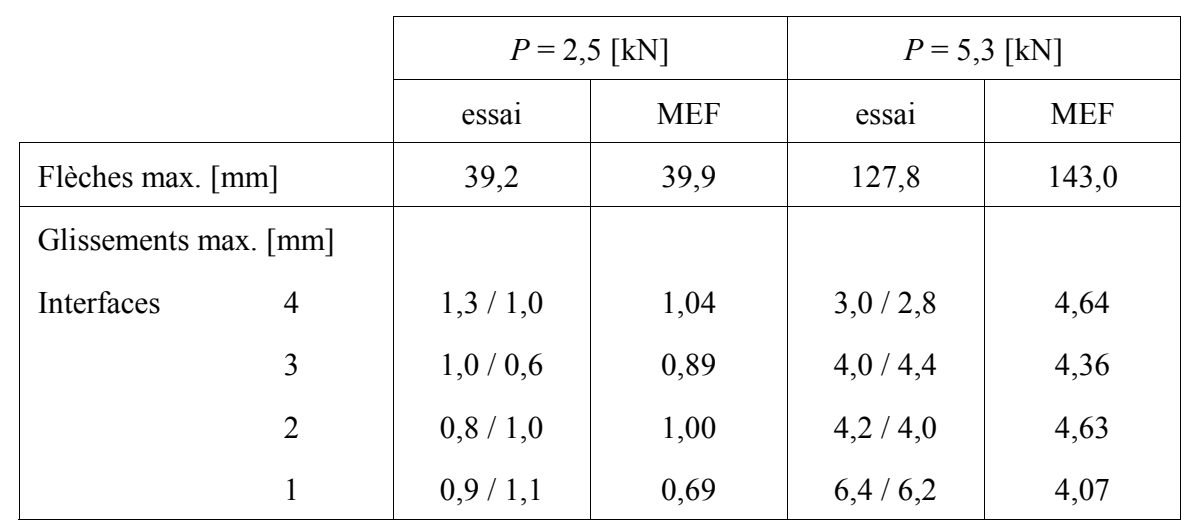

Tableau 5. Comparaison des flèches et des glissements

\section{Conclusions}

Cet article montre que, par un choix judicieux des inconnues cinématiques, on peut traiter avec succès le problème des poutres multicouches avec glissement aux interfaces dans le domaine géométriquement non linéaire (grands déplacements). On peut y greffer sans difficulté la non-linéarité matérielle tant des couches que des glissements d'interfaces.

Remerciements

Les auteurs remercient le Professeur Adnan Ibrahimbegović (LMT, ENS Cachan), avec lequel ils ont eu de fructueuses discussions. 
580 Revue européenne de mécanique numérique. Volume $16-\mathrm{n}^{\circ}$ 5/2007

\section{Bibliographie}

Batoz J.-L., Dhatt G., Modélisation des structures par éléments finis, Poutres et plaques, vol. 2, Hermès, Paris, 1990.

Belytschko T., Liu W. K., Moran B., Nonlinear finite elements for continua and structures, Wiley, 2000.

Carrera E., "Theories and finite elements for multilayered, anisotropic, composite plates and shells", Arch. Comput. Meth. Engng., vol. 9, n² 2, 2002, p. 87, Ibid., "Developments, ideas and evaluations based upon Reissner's variational theorem in the modelling of multilayered plates and shells", Applied Mech. Review, vol. 54, 2001.

Cas B., Saje M., Planinc I., "Nonlinear finite element analysis of composite planar frames with interlayer slip", Computers \& Structures, vol. 82, 2004, p. 1901-1912.

Crisfield M. A., Non-linear finite element analysis of solids and structures, vol. 1, Wiley 1991.

Engesser F., "Ueber verdübelte Balken”, Der Bauingenieur, Heft 8, 1922.

Fazio P., Hussein R. A. M., Ha K. H., "Sandwich beam-columns with interlayer slip", Eng. Mech. Div. ASCE, vol. 108, n EM2, 1982.

Frangi A., Fontana M., "Elasto-plastic model for timber-concrete composite beams with ductile connection", Structural Engineering International, n 1, 2003.

Frey F., Ibrahimbegovic A., Rebora B., Camacho A., Falla Luque C., Les verrouillages de l'élément de poutre de Bernoulli dans le cas plan, Rapports LSC 92/13, 95/07 et 96/06, LSC, EPFL, 1992/95/96.

Girhammar U. A., Gopu V. K. A., "Composite beam-columns with interlayer slip, Exact analysis”, J. Struct. Engng. ASCE, vol. 119, n 4, 1993.

Gollwitzer T., Gebbeken N., Ein neues FEM-Stabelement für nachgiebige Verbundquerschnitte, Bericht 03/3 aus dem Konstruktiven Ingenieurbau, Universität der Bundeswehr München, 2003.

Goodman J. R., Popov E. P., “Layered beam systems with interlayer slip”, J. Struct. Div. ASCE, vol. 94, n $\mathrm{ST} 11,1968$.

Heimeshoff B., "Zur Berechnung von Biegeträgern aus nachgiebig miteinander verbundenen Querschnittsteilen im Ingenieurholzbau”, holz als Roh- und Werkstoff, 45, 1987, p 237-241.

Hübner L., Holzflächentragwerte in Brettstapelbauweise, Diplomarbeit, IBOIS-EPFL, Lausanne, 2000.

Kamiya F., "Buckling of sheathed walls: nonlinear analysis", J. Struct. Engng. ASCE, vol. $114, \mathrm{n}^{\circ} 3,1987$.

Kapania R. K., Raciti S., "Recent advances in analysis of laminated beams and plates", AIAA $J .$, vol. 27, n $7,1989$.

Krawczyk P., Frey F., Zieliński A. P., Rebora B., "Large Deflections of Laminated Beams with Interlayer Slips”, Eng. Comp., 2006 (submitted). 
Kreuzinger H., Träger und Stützen aus nachgiebig verbundenen Querschnittsteilen, EUROFORTECH (EU Comett Programms), STEP B11 Bemessung und Baustoffe, HolzFachverlag, 1995.

Moehler K., Ueber das Tragverhalten von Biegeträgern und Druckstäben mit zusammengesetzten Querschnitten und nachgiebigen Verbindungsmitteln, Dissertation, TU Karlsruhe, 1956.

Natterer J., Hoeft M., Zum Tragverhalten von Holz-Beton-Verbundkonstruktionen, Forschungsbericht CERS Nr. 1345, EPFL, Lausanne, 1987.

Newmark N. M., Siess C. P., Viest I. M., "Tests and analysis of composite beams with incomplete interaction", SESA Proceedings, vol. 9, $\mathrm{n}^{\circ}$ 1, 1951.

Noor A. K., Burton W. S., Bert C. W., "Computational models for sandwich panels and shells", Applied Mech. Review, vol. 49, n 3, 1996, p. 155.

Pirazzi C., Zur Berechnung von Holzschalen in Brettrippenbauweise mit elastischem Verbundquerschnitt, Thèse de doctorat $n^{\circ}$ 3229, EPFL, Lausanne, 2005.

Pischl R., "Ein Beitrag zur Berechnung zusammengesetzter hölzerner Biegeträger", Der Bauingenieur, 43, 1968, Heft 12.

Richard R. M., A study of structural systems having nonlinear elements, PhD thesis, Purdue Univ., Lafayette, 1961.

Schelling W., Die Berechnung nachgiebig verbundener zusammengesetzter Biegeträger im Ingenieurholzbau, Dissertation, TU Karlsruhe, 1968.

StüssI F., "Beiträge zur Berechnung und Ausbildung zusammengesetzter Vollwandträger", Schweizerische Bauzeitung, Band 121, Nr. 9, S., 1943.

Stüssi F., Zusammengesetzte Vollwandträger Mémoires de l'AIPC, VIII, Zürich 1947.

Thompson E. G., Goodman J. R., VAnderbilt M. D., "Finite element analysis of layered wood systems", J. Struct. Div. ASCE, vol. 101, n ST12, 1975.

Vanderbilt M. D., Goodman J. R., Criswell M. E., "Service and overload behavior of wood joist floor systems", J. Struct. Div. ASCE, vol. 100, n ST1, 1974.

Wheat D. L., Calixto J. M., "Nonlinear analysis of two-layered wood members with interlayer slip", J. Struct. Engng. ASCE, vol. 120, n 6, 1994.

Yam L. C. P., Chapman J. C., "The inelastic behaviour of continuous composite beams of steel and concrete", Proc. Instn Civ. Engrs, vol. 53, 1972. 
